\title{
Work incentives and household insurance: Sequential contracting with altruistic individuals and moral hazard*
}

\author{
Cécile Aubert ${ }^{\dagger}$
}

\begin{abstract}
Two agents sequentially contracts with different principals under moral hazard. If agents care for one another, the second principal gains by insuring them over first wages. Even with independent tasks, the first principal must offer riskier payments to induce effort.
\end{abstract}

JEL Codes: D10, D64, D82.

Keywords: Incentives, Altruism, Moral Hazard.

\section{Introduction}

The interplay between altruism and incentives has been the object of many studies since the seminal papers by Becker $(1974,1976,1991)$ and Buchanan (1975). Most papers focus on incentives between two individuals, one of which (at least) is altruistic with respect to the other. Yet the existence of altruism between individuals may also affect their incentives with respect to a third party who is not altruistic nor the object of altruism. Consider a moral hazard setting in which two individuals are hired by different principals; if these individuals care for

\footnotetext{
${ }^{*}$ I am very grateful to Jean-Jacques Laffont $\dagger$ for helpful discussions on this topic. I also thank François Salanié for very helpful comments, and seminar participants in Cambridge, Leicester, and Toulouse.

${ }^{\dagger}$ Eurisco, Université Paris Dauphine. Address: Département Economie Appliquée, Université Paris Dauphine, place de Lattre de Tassigny, F - 75016 Paris, France. Tel: (33)1 440545 23. Fax: (33)1 44054733 . E-mail address: Cecile.Aubert@dauphine.fr
} 
one another, their ability to insure each other modifies incentive costs. Household members may be such agents, especially if one obtains a position before the other does, and employers are aware of this fact. Such 'small' employment markets may especially be found in rural, possibly developing, areas or in small communities.

We consider such a setting where two agents are altruistic with respect to each other, and choose to share their resources. Each is engaged in a contractual relationship with a riskneutral principal, under moral hazard. The individuals are not altruistic with respect neither to their principal nor to the task they undertake (contrary to the literature on non-profits or on physicians $\left.^{1}\right)$. Outcomes are learned sequentially. Tasks are assumed to be perfectly independent. Yet the fact that altruistic agents share resources can be exploited by the second principal - the one for which performance is known second: this principal will indeed find it optimal to make her wages depend on both outcomes, as both determines her agent's revenues. She will thus (at least partly) insure her agent against first-income variations. Altruism creates a situation similar to common agency with moral hazard ${ }^{2}$, as in Tommasi and Weinschelbaum (2004). The first principal must take into account the best response of the second principal; she must offer steeper contracts to induce effort despite indirect insurance through the second agent.

\section{The model}

The players Two individuals, $A$ and $B$, are altruistic with respect to each other. To simplify and avoid making assumptions on how agents solve disagreements on resource sharing, we assume

\footnotetext{
${ }^{1}$ This literature has mostly considered settings in which agents are intrinsically motivated by the task they undertake: Choné and Ma (2004) consider physicians' private information on their altruism with respect to patients, and on the latters' health; Francois (2003) and Rowat and Seabright (2005) analyze effort incentives for motivated employees in NGOs. Our setting strongly differs as there is no intrinsic motivation, and principals act strategically with respect to one another, something that cannot arise in these other papers.

${ }^{2}$ See Arnott and Stiglitz (1991) on self-insurance, and Bizer and DeMarzo (1992) and Bisin and Guaitoli (2004) on multi-contracting in banking.
} 
that altruism appears in an additive separable way and that both agents have the same degree of altruism. Their utility function is thus given by a 'von Neumann-Morgenstern'-type bi-variate function $U^{i}\left(t, U^{j}\right)=u(t)+U^{j}, i \neq j, i, j=1,2$, where $u($.$) is a strictly concave and increasing$ function, with $u(0)$ normalized to zero. The individuals will thus always prefer to share equally total resources. ${ }^{3}$

Each individual contracts with a different, risk-neutral, principal: principal $P^{A}$ hires individual $A$ to exerts some unobservable effort $e^{a}$ in some task $a$, in exchange for some transfer $t^{A}$, and $P^{B}$ does the same with individual $B$. Project $a$ yields a verifiable return, $S^{a}>0$ in case of success and 0 in case of failure. Effort $e^{a}$ is discrete (0 or 1$)$ and non observable. If agent $A$ exerts a high effort, $e^{a}=1$, he incurs a (non observable) disutility of effort $\psi$ and the probability that the project is successful is $p_{1}$. If he shirks on the other hand, and exerts effort $e=0$ only, he incurs no disutility but the probability of success falls to $p_{0}<p_{1}$.

To simplify, we assume that principal $P^{B}$ does not wish to induce effort in task $b$. She thus does not need to make payments depend on her agent's performance (a null transfer would be optimal if she was facing a non-altruistic agent). Hence, performance in this task can be assumed to be certain, $\bar{S}^{b}$. The analysis is similar, but expressions become more complicated, if $P^{B}$ also requires an effort to increase the probability of a high performance in task $b$, as four different states of nature, and additional incentive constraints, must be considered.

The two projects are completely independent, yet due to altruism, the transfer given by one principal affects the utility level obtained by the agent of the other principal: altruism creates an indirect externality between principals. Once inter-agent transfers are taken into account, the setting becomes formally similar to a sequential common agency game with a single agent.

\footnotetext{
${ }^{3}$ Full insurance of one individual here implies into full insurance of the other. This is a restriction, but it allows highlighting the interlinkage between incentive contracts for independent tasks due to altruism. It should become clear that our qualitative results would remain valid with less altruism.
} 
The timing Principal $P^{A}$ must pay her agent first and cannot condition transfers on $B$ 's wage or contract. The timing ${ }^{4}$ is the following:

1. Principal $P^{A}$ offers a contract to agent $A$, who accepts or refuses it.

2. Principal $P^{B}$ then offers a contract to $B$, who accepts or refuses it.

3. If agent $A$ has accepted his contract, he privately chooses effort $e^{a}$.

4. The outcome for task $a$ is observed, and $t^{A}$ is publicly paid by $P^{A}$.

5. Agent $B$ is paid by $P^{B}$, and the total payment is shared by $B$ and $A$.

A benchmark Assume that the two agents are selfish, and cannot insure each other. Then principal $P^{a}$ solves a standard moral hazard game by having both the participation and the incentive compatibility constraint of her agent binding. She offers transfers $\bar{t}^{a}$ in case of success and $\underline{t}^{a}$ in case of failure, where $u\left(\bar{t}^{a}\right)=\frac{1-p_{0}}{p_{1}-p_{0}} \psi$ and $u\left(\underline{t}^{a}\right)=-\frac{p_{0}}{p_{1}-p_{0}} \psi$.

\section{The second-stage contract}

If agent $A$ has refused the contract offered by principal $P^{A}$, the problem is standard, except that a transfer $t^{B}$ yields utility $2 u\left(\frac{t^{B}}{2}\right)$ to the agent. Since $u($.$) is strictly concave, 2 u\left(\frac{x}{2}\right)>u(x)$, and $P^{B}$ benefits from altruism. The optimal contract gives a null expected utility to agents $B$ and $A$.

Assume on the other hand that $A$ has accepted the contract offer, and suppose for the moment that principal $P^{A}$ prefers to induce effort from her agent. She pays him $\bar{t}^{A}$ in case of success, and $\underline{t}^{A}$ in case of failure. Principal $P^{B}$ can observe and contract on the outcome of task

\footnotetext{
${ }^{4}$ Since the contract offered by $P^{A}$ is accepted or refused before principal $P^{B}$ makes an offer to $B, P^{A}$ cannot take advantage of the subsequent relationship between agent $B$ and $P^{B}$ in his dealings with $A$.
} 
a. Although tasks are independent, she should offer transfers $t^{B}$ that depend on whether agent $A$ has succeeded (and obtained a high transfer $\bar{t}^{a}$ ) or not. ${ }^{5}$

One might think that making the transfer depend on an unrelated factor increases the risk the agent bears. However, principal $P^{B}$ can use transfers that are differentiated according to performance in the other task so as to insure her agent, thereby reducing expected transfers for this agent. This allows the risk-neutral principal to offer negative expected transfers to her agent, transfers that are nevertheless accepted.

The expected utility of individual $B$ is indeed given by

$$
p_{e^{a}} u\left(\frac{\bar{t}^{A}+t^{B}\left(\bar{t}^{A}\right)}{2}\right)+\left(1-p_{e^{a}}\right) u\left(\frac{\underline{t}^{A}+t^{B}\left(\underline{t}^{A}\right)}{2}\right) .
$$

Let us denote by $T^{B}\left(e^{a}\right)$ the certainty equivalent that gives him the same utility as when $t^{B}\left(\bar{t}^{A}\right)=t^{B}\left(\underline{t}^{A}\right)=0$ for effort $e^{a}$ in task $a$. From the strict concavity of $u(),. T^{B}\left(e^{a}\right)<$ $p_{e^{a}} u^{-1}\left(\frac{\bar{t}^{A}}{2}\right)+\left(1-p_{e^{a}}\right) u^{-1}\left(\frac{t^{A}}{2}\right)$. Principal $P^{B}$ can thus make profits by offering agent $B$ transfers that compensate for the payments of the other principal so as to ensure that the agent always obtains the certainty equivalent: $t^{B}\left(\bar{t}^{A}\right)=T^{B}\left(e^{a}\right)-\bar{t}^{A}$ and $t^{B}\left(\underline{t}^{A}\right)=T^{B}\left(e^{a}\right)-\underline{t}^{A}$. Fully insuring her agent is thus always profitable for the second principal. The result holds for any given effort level $e^{a}$ by individual $A$.

Lemma 1 Due to agents' altruism for one another, the second principal finds it optimal to condition payments on the outcome of the first contract even with independent tasks and no direct externalities.

There exists a potential conflict of interests between the two principals since the second

\footnotetext{
${ }^{5}$ If performance in task $b$ was also random, and depended on some effort $e^{b}$, principal $P^{B}$ should offer four different transfers, $t_{a b}^{B}$ when both tasks succeed, $t_{a}^{B}$ when only task $B$ succeeds, similarly $t_{b}^{B}$ when only $A$ succeeds, and $t_{\emptyset}^{B}$ when both tasks fail. Separating between four states of nature allows insuring agent $B$ against income shocks that do not depend on his effort $e^{b}$, and obtaining effort at a lower cost.
} 
principal can exploit her agent's risk aversion to her advantage.

Given that the agents equally share their resources, when agent $B$ is perfectly ${ }^{6}$ insured, so is agent $a$. The latter no longer has incentives to exert effort, and chooses $e^{a}=0$.

This analysis is not sufficient, though, as the second principal may find it even more profitable not to fully insure her agent, so as to still induce effort from $A$, by satisfying an incentive compatibility constraint. The incentives of the second principal depend on the variability of the contract initially offered, as we discuss below.

\section{The first-stage contract}

- Let us first consider the situation in which the first principal, $P^{A}$, offers a contract that is not steep enough to make it profitable for the second principal to induce effort in task $a$. As the agent will not exert effort, principal $P^{a}$ will offer a fixed, null, transfer to her agent. Principal $P^{B}$ then offers the same contract as with a selfish agent ${ }^{7}$. Although the transfers received by agent $B$ ex post do not depend on the outcome in task $a$, the possibility that $P^{B}$ differentiates transfers according to more states imply that it can counter the incentive scheme designed by the first principal and induce no effort, as long as these transfers are not steep enough.

- Assume now that principal $P^{A}$ offer a wage scheme sufficiently risky to ensure that the second principal prefers to still induce effort in the first task (this scheme is characterized below). When contracting with $B$, the second principal will have the incentive compatibility constraint with respect to task $a$ bind, to limit incentive costs:

$$
\left(p_{1}-p_{0}\right)\left[u\left(\frac{\bar{t}^{A}+t^{B}\left(\bar{t}^{A}\right)}{2}\right)-u\left(\frac{\underline{t}^{A}+t^{B}\left(\underline{t}^{A}\right)}{2}\right)\right]=\psi .
$$

\footnotetext{
${ }^{6}$ In a more general model in which sharing would be incomplete, agent $A$ might not be perfectly insured when $B$ is, but it may still be the case that his incentive compatibility constraint be violated due to insurance by principal $P^{B}$.

${ }^{7}$ It would not, obviously, be an equilibrium if the game was simultaneous.
} 
As the participation constraint will also bind, we obtain as usual that $u\left(\frac{\bar{t}^{A}+t^{B}\left(\bar{t}^{A}\right)}{2}\right)=\frac{1-p_{0}}{p_{1}-p_{0}} \psi$ and $u\left(\frac{\underline{t}^{A}+t^{B}\left(\underline{t}^{A}\right)}{2}\right)=-\frac{p_{0}}{p_{1}-p_{0}} \psi$. The gain obtained by $P^{B}$ will be

$$
p_{1}\left[\bar{t}^{A}-u^{-1}\left(\frac{1-p_{0}}{p_{1}-p_{0}} \psi\right)\right]+\left(1-p_{1}\right)\left[\underline{t}^{A}-u^{-1}\left(-\frac{p_{0}}{p_{1}-p_{0}} \psi\right)\right] .
$$

The second principal will prefer to induce effort from the agent if and only if this expected gain is larger than the expected gain when fully insuring the agent, i.e.,

$$
p_{1} \bar{t}^{A}+\left(1-p_{1}\right) \underline{t}^{A}-u^{-1}\left(p_{1} u\left(\frac{\bar{t}^{A}}{2}\right)+\left(1-p_{1}\right) u\left(\frac{\underline{t}^{A}}{2}\right)\right) .
$$

If the first principal wants to obtain effort is task $a$, she must satisfy an incentive compatibility constraint with respect to the second principal. This constraint is

$$
p_{1} u\left(\frac{\bar{t}^{A}}{2}\right)+\left(1-p_{1}\right) u\left(\frac{\underline{t}^{A}}{2}\right) \geq u\left(p_{1} u^{-1}\left(\frac{1-p_{0}}{p_{1}-p_{0}} \psi\right)+\left(1-p_{1}\right) u^{-1}\left(-\frac{p_{0}}{p_{1}-p_{0}} \psi\right)\right),
$$

and replaces the incentive compatibility constraint with respect to agent $A$. The first contract must thus be riskier.

The first principal also faces a participation constraint with respect to principal $P^{B}$ :

$$
p_{1}\left[\bar{t}^{A}-u^{-1}\left(\frac{1-p_{0}}{p_{1}-p_{0}} \psi\right)\right]+\left(1-p_{1}\right)\left[\underline{t}^{A}-u^{-1}\left(-\frac{p_{0}}{p_{1}-p_{0}} \psi\right)\right] \geq 0 .
$$

Because incentive constraints are changed with respect to the benchmark situation of a selfish agent, the costs of inducing effort are modified. The first principal will therefore less often prefer to induce a high effort.

Proposition 1 Assume that contracts and outcomes are public, and that principals sequentially contract with agents who are altruistic vis-à-vis each other. The principal who acts first must offer riskier contracts to induce effort in equilibrium, despite subsequent contracting by the second principal. A high effort level will less often be induced. 


\section{Conclusion}

Our setting, although specific, highlights interesting insurance effects of altruism. If the two principals were acting simultaneously, the game would be formally similar (once intra-household transfers are taken into account) to that in Bernheim and Whinston (1986) but with delegated, common agency. In both settings, the equilibrium payment is the minimum one necessary to obtain the effort level required. And in both, equilibria can be interpreted as resulting from the delegation by one principal of her task to the other principal, via the contract offered to the agent. Here however, there is a single equilibrium and the identity of the principal who delegates is no longer undetermined. If outcomes were private, our results would be more drastically affected: the second principal would face adverse selection regarding the transfer paid to agent $A$. As the second principal's transfer is a decreasing function of the first, agents would always underestimate $t^{A}$ when they have CARA preferences: the second principal could not offer insurance. Our setting is more relevant for 'small' employment markets, in which more information is available, as mentioned in the introduction.

The externality created between principals by altruism remains striking: Even if no insurer is available to the first agent, insurance by his mate, possibly via another work contract, strongly affects effort incentives. Recognizing the role of the environment (including the affective environment) of the agent is thus essential. Our results may apply to situations in which an individual obtains a position after his/her spouse, and the second employer is aware of the insurance properties of the second employment - married women employment decisions, in particular, are often partly based on insurance arguments.

In such a context, a potential solution for the first employer to restore incentives at a lower cost is to use non monetary benefits that are more difficult to share - free or subsidized lunches, 
transportation to the work place, or health benefits.

\section{References}

Arnott, R., and J. E. Stiglitz, 1991, Moral Hazard and Nonmarket Institutions: Dysfunctional Crowding Out of Peer Monitoring?, The American Economic Review 81: 179-190.

Becker, G., 1974, A Theory of Social Interaction, Journal of Political Economy 82: 1063-1093.

Becker, G., 1976, Altruism, Egoism, and Genetic Fitness: Economics and Sociobiology, Journal of Economic Literature 14: 817-826.

Becker, G., 1991, A Treatise on the Family (Harvard University Press, Cambridge, MA).

Bernheim, D., and M. Whinston, 1986, Common Agency, Econometrica 54: 923942.

Bisin, A., and D. Guaitoli, 2004, Moral Hazard and Nonexclusive Contracts Production: Theories and Evidence from Joint Ventures, The RAND Journal of Economics 35: 306-328.

Bizer, D., and P. M. DeMarzo, 1992, Sequential Banking, The Journal of Political Economy 100: $41-61$.

Buchanan, J., 1975, The Samaritan's Dilemma, in E. Phelps, ed., Altruism, Morality, and Economic Theory (Sage Foundation, New York, NY).

Choné, P., and C-T. A. Ma, 2004, Asymmetric Information from Physician Agency: Optimal Payment and Healthcare Quantity, mimeo, Crest-Lei and Boston University.

Francois, P., 2003, Not-for-Profit Provision of Public Services, Economic Journal 115: 53-61.

Rowat, C., and P. Seabright, 2005, Intermediation by Aid Agencies, Journal of Development Economics forthcoming.

Tommasi, M., and F. Weinschelbaum, 2004, Principal-Agent Contracts under the Threat of Insurance, mimeo, Universidad de San Andrés. 